\title{
Higher-Order Vagueness and the Vague- ness of 'Vague'
}

Achille C. VARZI

Sorensen (1985) has argued that 'vague' is itself a vague predicate; it is just as sorites-prone as its positive instances. This result has been exploited by Hyde (1994) in an ingenious attempt to establish that vague predicates must necessarily suffer from higher-order vagueness. More precisely, Hyde has argued that the vagueness of 'vague' ensures that the 'paradigmatic conception', according to which predicate vagueness is characterized by the presence of border cases, need not be revised or further elaborated upon in order to account for the phenomenon of higher-order vagueness: if a predicate has border cases, it has border border cases. Tye (1994) has objected (convincingly I think) that this is too strong: all that follows from Sorensen's result is that there are some border border cases, but not necessarily border border cases of every vague predicate. I shall argue that this is still too strong: Sorensen's proof presupposes the existence of border border cases, hence cannot be used to establish that fact on pain of circularity.

\section{1.}

Sorensen's argument for the vagueness of 'vague' goes as follows. First, vague predicates give rise to the sorites paradox. For instance, the vagueness of 'small' allows one to construct the following soritical argument, in which a false conclusion is inferred from two seemingly true premisses:

(1a) 1 is small

(1b) For every integer $n$ : if $n$ is small, then so is $n+1$.

(1c) Therefore, $10^{10}$ is small.

Now, for every integer $n$, let ' $n$-small' be a numerical predicate defined by the following condition: 
(2) $k$ is $n$-small iff $k$ is either small or less than $n$.

Clearly, ' 1 -small' is just as vague as 'small': both apply to o and apply exactly in the same way to all other integers. By contrast, when $n$ is clearly not small, say $n=10^{10}$, then the extension of the predicate ' $n$ small' is determined exclusively by the 'less than $n$ ' clause, and is therefore perfectly sharp: every integer less than $10^{10}$ is $10^{10}$-small, every other integer is not. Since there is no clear value of $n$ which marks the difference between predicates of the first sort (with border cases) and predicates of the second sort (without border cases), one can now construct a soritical argument for 'vague' that parallels (1a)-(1c):

(3a) ' 1 -small' is vague.

(3b) For every integer $n$ : if ' $n$-small' is vague, then so is ' $n+1$-small'.

(3c) Therefore ' $10^{10}$-small' is vague.

Consequently, 'vague' is vague.

Some commentators (for instance Deas 1989) have objected to this line of reasoning on account of its parasitic form. After all, the vagueness exhibited by (3a)-(3c) is really not a feature of 'vague' butindirectly - a feature of 'small'. 'This is a fair remark. However it is hardly a relevant objection to Sorensen's argument: certainly 'married to a bald man' is truly a vague predicate, even though its vagueness is entirely parasitic upon that of 'bald'. At most, one can object to Sorensen that his sorites series for 'vague' involves a class of very special and somewhat artificial predicates. But this is an ad hoc manoeuvre. If such predicates are accepted, then Sorensen's argument is perfectly sound.

\section{2.}

Let us accept such predicates. Does Sorensen's result support the claim that there exist predicates that suffer from higher-order vagueness? Hyde has argued that it does:

I think that there are higher orders of vagueness, but that this is already entailed by the paradigmatic conception and can be seen to follow when the

\footnotetext{
${ }^{1}$ Deas grounds his argument on the observation that (2) logically implies that ' $n$-small' is vague iff $n$ is small. But this is mistaken. The left-to-right direction of this biconditional can be resisted by letting $n$ be a border case of 'small'. For then $n$ is also a border case for 'small or less than $n$ ', hence a border case for ' $n$-small'. In other words, ' $n$-small' can be vague even when $n$ is not small (but, rather, a border case for 'small').
} 
notion of 'border case' employed therein is properly understood. (Hyde 1994, p. 39)

In a nutshell, the argument is that on the paradigmatic conception the vagueness of 'vague' entails the existence of border border cases in just the same way that the vagueness of 'small' entails the existence of border cases for 'small'. Here is a more detailed reconstruction of this line of reasoning:

(4a) On the paradigmatic conception, '... is vague' can be analysed as 'there are border cases of ....'

(4b) The predicate 'vague' is vague.

(4c) Hence, on the paradigmatic conception 'there are border cases of ...' is vague (from (4a) and (4b)).

(4d) The quantifier 'there are ...' is not vague.

(4e) Hence, on the paradigmatic conception 'border case of ...' is vague (from $(4 \mathrm{c})$ and $(4 \mathrm{~d})$ ).

(4f) Hence, on the paradigmatic conception there are border cases of 'border case of ...' (from (4a) and (4e)).

(4g) Hence, on the paradigmatic conception there are predicates that have border border cases (from (4f)).

There is no question that this argument is valid. Thus, as long as we accept (4b) and (4d), Hyde's reasoning would seem to establish that the 'paradigmatic conception' implies the existence of some cases of higher-order vagueness. (As I mentioned, Hyde's original argument attempts to establish a stronger result, namely, that every vague predicate has border border cases. The present reconstruction takes Tye's objection into account.)

I do not wish to quarrel premiss (4d) (though some might want to do just that). And, of course, premiss (4b) is precisely the upshot of Sorensen's argument. So is Hyde's reasoning sound? It may well be (and personally I think it is). The trouble is that in this context we cannot rely on Sorensen's argument to motivate (4b), for that argument presupposes the existence of border border cases. Specifically, it presupposes the existence of border border cases for 'small'. To see this, notice first that (2) equates the extension of ' $n$-small' with that of 'either small or less than $n$ '. It follows that these two predicates have the same border cases - that is, 
(5) $k$ is a border case of ' $n$-small' iff $k$ is a border case of 'either small or less than $n$ ?

Since 'less than $n$ ' is precise for all $n$, this amounts to

(6) $k$ is a border case of ' $n$-small' iff $k$ is a border case of 'small' and greater than or equal to $n^{2}$

And since every number is greater than or equal to itself, this in turn implies

(7) $k$ is a border case of ' $k$-small' iff $k$ is a border case of 'small'.

Now, suppose that 'small' does not have border border cases: every integer is either a positive case, or a negative case, or a border case of 'small'. Suppose $k$ is the cut-off point between the border cases and the negative cases: $k$ is a border case of 'small', but $k+1$ is definitely not small. Then, by ( 7$), k$ is a border case of ' $k$-small', which means that ' $k$ small' is vague. But no number is a border case of ' $k+1$-small', since (i) every number less than or equal to $k$ is less than $k+1$, hence definitely $k+1$-small, and (ii) every number greater than $k$ is definitely not small (by assumption) and definitely not less than $k+1$, hence definitely not $k+1$-small. So ' $k+1$-small' is not vague. So there is some value of $n$ (namely $k$ ) such that ' $n$-small' is vague but ' $n+1$-small' is not vague. And this contradicts the inductive premiss ( $3 b$ ) of the sorites argument for 'vague'.

Of course, this is no objection to Sorensen's argument, because Sorensen is perfectly entitled to assume that 'small' has border border cases. (In a way, this is reflected in the inductive premiss ( $1 \mathrm{~b}$ ) of the sorites argument for 'small', though we need not commit ourselves to this way of characterizing higher-order vagueness.) But in the context of Hyde's argument we are not entitled to that assumption, on pain of circularity. Thus, for the purpose of that argument Sorensen's affidavit of premiss ( $4 \mathrm{~b})$ is unreliable: this premiss must be independently established. Or it must be granted as obvious, as Austin (1962: 126f) suggested. ${ }^{3}$

\footnotetext{
${ }^{2}$ At least, this is true on any semantics for 'or' that makes a disjunction true as long as one of its disjuncts is true (even though the other disjunct is indeterminate).

${ }^{3}$ Thanks to Haim Gaifman and Dominic Hyde for helpful discussion and exchanges.
} 


\section{References}

Austin, J. L., 1962: Sense and Sensibilia, ed. G. J. Warnock, Oxford: Oxford University Press.

Deas, R. 1989: 'Sorensen's Sorites', Analysis 49, pp. 26-31.

Hyde, D. 1994: 'Why Higher-Order Vagueness is a Pseudo-Problem', Mind 103, pp. 35-41.

Sorensen, R. A. 1985: 'An Argument for the Vagueness of "Vague", Analysis 27, pp. 134-37.

Tye, M. 1994: 'Why the Vague Need Not be Higher-Order Vague', Mind 103, pp. 42-44. 

Copyright of Mind is the property of The Mind Association and its content may not be copied or emailed to multiple sites or posted to a listserv without the copyright holder's express written permission. However, users may print, download, or email articles for individual use. 